\title{
An entrancing tale of cross-disciplinary bridge building and burning in ethnopsychophysiomusicology
}

\author{
PETR JANATA \\ University of California, Davis [1]
}

\begin{abstract}
Having a paper accepted for publication is challenging, even under the best of circumstances, as when reporting an incremental finding in a field that is one's home discipline. The process becomes considerably more difficult when venturing into foreign disciplines in which methodological conventions and assumptions may differ from those one is familiar with. Provocative topics may further exacerbate the reticence of reviewers and editors to welcome cross-disciplinary research to a journal's pages. Here, a pair of papers, one of which describes a study of possible physiological correlates of musicinduced trance states, and the other which describes the challenging journey to get the research performed and published, provide a case study for examining whether epistemological divides can be bridged in the face of editorial obstinacy.
\end{abstract}

Submitted 2009 May 7; accepted 2009 June 3.

KEYWORDS: editorial review, epistemology, bias, music, emotion

A pair of papers in this issue (Becker, 2009; Penman \& Becker, 2009) provides a rare public glimpse into the harrowing drama that often plays itself out in academic circles when scholars seek to publish their work. There are two stories here, each meriting individual commentary. The first is the research contribution describing changes in physiological states as individuals prone to trancing listen to personally salient selections of music (Penman \& Becker, 2009). The second is the tale of an ethnomusicologist failing to get the research published in the Psychology of Music (Becker, 2009).

Ostensibly the problem is one of a fundamental incompatibility between "science" and "humanities" research and the attendant difficulty in finding suitable publication outlets for research projects that bridge the two realms. Becker nicely summarizes some of the expectations and characteristics of inquiry in each realm in her prologue, and I find that the introduction of the research article respects the different scholarly traditions that are being combined. Although it might be an alluring scholarly exercise to dissect and compare the standards of scholarship within the "scientific" and "humanistic" realms, as a way of reaffirming some endemic incompatibility, I think the challenges confronting the publication of interdisciplinary work have more to do with human psychology than epistemological hurdles. Specifically, we need to recognize that editorial decisions, on the part of reviewers and editors, are likely shaped as much by emotional states associated with personal prejudices and editorial demand characteristics, or even the time of day that the review is performed, as they are by objective and immutable scholarship criteria that are immune to ignorance or disciplinary or personal biases (Abramowitz, Gomes, \& Abramowitz, 1975; Morton, Haslam, Postmes, \& Ryan, 2006; Peters \& Ceci, 1982).

\section{RESPONSIBILITY IN THE REVIEW PROCESS}

Assuming that the publication of papers on provocative cross-disciplinary topics is a worthy pursuit for strengthening the overall web of human understanding, what are the responsibilities of authors, editors, and reviewers when confronted with manuscripts that push the comfort zones and boundaries of different disciplines and styles of scholarship? What is necessary for meritorious ideas and discourse to surface while each discipline's sense of academic integrity is maintained? Personally I do not believe that standards should be relaxed for the sake of promoting cross-disciplinary publication; as an author I have a responsibility to present and revise my work so that the standards demanded by the editor and reviewers are met. The role of the editor is perhaps the most challenging when adjudicating and managing the review process for 
crosscutting work. An editor should arguably be a champion for provocative manuscripts, as these are more likely to garner readers and stimulate further work than are manuscripts that represent yet another variation on a common theme. However, provocative ideas that are not in accord with one's world view are likely to meet with increased resistance (cognitive dissonance) on the part of the reviewers, with the effect of increased probability of disciplinary prejudices influencing reviewers' comments, resulting in negative recommendations or less enthusiastic endorsements (Abramowitz et al., 1975). A reviewer may feel rigorous and righteous about recommending rejection if a particular experimental approach does not conform to his or her (challenged) belief system, but the editor does not, in principle, have that luxury. When adjudicating a review, the editor must determine whether the demands of a reviewer are reasonable and whether the criticisms are truly damning, or only appear as fatal flaws if a particular, and perhaps imprudently narrow perspective is assumed. Having spent my professional life in the neural and psychological sciences, I find that relatively few perspectives have been established so thoroughly as to be unassailable - especially those at the cutting edge. This includes the topics of emotional and physiological responses to music.

\section{REVIEWER COMMENTS}

I would be playing ivory-tower games if I pretended that I believe the failure of Penman and Becker's paper to make it onto the pages of Psychology of Music was due to irreconcilable differences between the ways that science and humanities do things. In my opinion, it was a failure of the review process. Below, I provide two sets of brief comments [2]. One is from the perspective of a reviewer assessing the manuscript, i.e. what are the substantive issues that I would like to see addressed in a revision. The other set is from the perspective of a reviewer assessing the comments of other reviewers and the three categories of criticisms identified by Becker.

\section{A "scientific" reviewer perspective}

Overall, I found Penman and Becker's paper to be well written and the principle findings that the GSR response and heart rate variability are significantly and uniquely increased in Pentecostal Ecstatics listening to their self-selected music relative to control music to be indisputable. Becker embarked on the research with a clearly defined hypothesis, designed an adequate experiment to test the hypothesis, obtained a statistically significant result that supported her hypothesis, and reported as much. A variety of control groups went through the same procedure, and similar effects on autonomic nervous system responses were not observed. Irrespective of what one believes about physiological responses to music, trance states, or the relationship of music to trance states, the basic result seems clean and its relationship to the psychology of music clearly relevant. A substantial effort was made to orient the reader to the different approaches with which one might tackle the issue of music and trance, and to identify the shortcomings of each approach.

I did find myself with several methodological concerns that I would have liked to see addressed. First, it was unclear how the second piece of control music was determined for each subject. It is stated that it was another subject's favorite piece, but how was the assignment for each subject determined or varied across subject groups in a way that differential biasing across groups could be ruled out? A second methodological concern pertains to the Intensity Level rating for each piece. Apparently, this is a subjective measurement scale devised by the authors based on several features of music. Though we are provided with examples of musical pieces characterizing each of the 9 intensity levels, and we are assured that both authors have extensive musical backgrounds, insufficient information is provided for a reader to really understand how an intensity score is derived. If I were to try to follow their heuristic to obtain an Intensity Score for an excerpt of music, I would be unable.

As a reviewer, I might also advise a slightly different approach to the statistical testing of the GSR effects. A two-factor ANOVA with Group and Music as factors, the latter a within-subjects factor, would be appropriate. This would allow for a test of a main effect of Music (self-selected versus control music), which would be expected given the literature cited. The test for a Group main effect would establish whether there were differences between groups in overall GSR. [A one-way ANOVA was used to test for group differences in responses to self-selected music, but the reported degrees of freedom do not make sense.] Finally, the Group X Music interaction could be tested which, given the plotted data, would likely show a significant effect. A follow-up t-test of responses to self-selected and control music within the group of Pentecostal 
Ecstatics would likely reveal this as the source of the interaction. As it was, this point was made in Figure 3 and the associated ANOVA performed on the (self-control music) difference scores, together with the follow-up pairwise comparisons.

While I'm not entirely convinced about the conclusion that Deep Listeners have stronger responses to music they love than do other groups (aside from the Pentecostal Ecstatics), because they fail to show a difference relative to control music and because they showed by far the greatest variability in responses, I found no "fatal flaws" in the paper that would preclude the primary and robust finding pertaining to the responses of the Pentecostal Ecstatics from being published. I would have recommended "Revise and Resubmit." The paper makes a novel contribution to our understanding of physiological responses to music and it raises the interesting question of whether autonomic nervous system activation is a prerequisite for entering a trance state.

\section{Reviewing the reviews}

Although it is not entirely proper, absent the full evidence, to decry as unfair the review process that Penman and Becker's manuscript was subjected to at Psychology of Music, the examples Becker provides of the three categories of critiques that she felt unable to address suggest that intervention on the part of the reviewing editor would have been appropriate. I find the spontaneous eruption of dialog between reviewers, with one of them emphatically suggesting that the other's apparently steadfast criticisms were unduly harsh given extensive precedent in the literature, to be telling of a review process gone awry.

Similarly, a reviewer's apparent insistence on the primacy of structural features in music as determinants of emotional responses to music, as opposed to personal associations with the music, belies a myopic view. Briefly considering that there is tremendous heterogeneity in genre preferences across individuals quickly forces one to concede, whether one is comfortable with the idea or not, that structural properties of music are not universal determinants of emotional response to music. If they were, how could it be the case that one person passionately likes while another passionately dislikes the same piece of music (assuming neither has a personal association with the particular piece)? The relative contributions of different triggers of emotional responses to music are a current topic of discussion (Juslin \& Västfjall, 2008). It seems inappropriate to cut down a paper because it doesn't reinforce one's personal view of how music shapes emotions. Moreover, assuming that the control pieces of music were distributed appropriately across the groups, any variance introduced by the structural differences in the pieces should have impacted the experimental and control groups similarly, so the overall experimental design renders the concern moot. Given that neither the sources of emotion in music nor the relationship of autonomic nervous system activity to emotion are settled matters in the broader literature, one hopes that this paper's publication was not derailed because an editor allowed reviewer recommendations stemming from such concerns to prevail (perhaps as a convenient way of deferring responsibility for his/her own discomfort with the paper's content).

Cross-disciplinary research is difficult, and as one of the reviewers wrote, "the problems of getting a genuine and solid experimental handle on the study of religious trance seem formidable." This type of statement raises several questions. First, should formidable questions go unanswered? If not, what are appropriate increments in which formidable challenges can be addressed? Should the fact that the topic of music and trance appears formidable somehow discount a statistically significant increase in ANS responsiveness that appears selective to a sample of individuals that is prone to trance? As reviewers and editors, do we feel better knowing that in rejecting a paper we have upheld rather than chipped away at an edifice of formidable challenge?

\section{NOTES}

[1] Correspondence regarding this manuscript should be addressed to

Petr Janata

Center for Mind and Brain

University of California, Davis

267 Cousteau Place

Davis, CA 95616

e-mail: pjanata@ucdavis.edu 
[2] I was not privy to any of the interchanges that occurred surrounding the Psychology of Music submission; my comments are based on the material presented in the target articles. I recognize that not all sides of the argument are represented in the introductory article (Becker, 2009), and I also disclose that Judith Becker and I have a collaboration in which members of her study group of Pentecostals are involved.

\section{REFERENCES}

Abramowitz, S. I., Gomes, B., \& Abramowitz, C. V. (1975). Publish or Politic: Referee Bias in Manuscript Review. Journal of Applied Social Psychology, 5(3): 187-200.

Becker, J. (2009). Crossing Boundaries. Empirical Musicology Review.

Juslin, P. N., \& Västfjall, D. (2008). Emotional responses to music: The need to consider underlying mechanisms. Behavioral and Brain Sciences, 31(5): 5559-5575.

Morton, T. A., Haslam, S. A., Postmes, T., \& Ryan, M. K. (2006). We value what values us: The appeal of identity-affirming science. Political Psychology, 27(6): 823-838.

Penman, J., \& Becker, J. (2009). Religious ecstatics, "deep listeners," and musical emotion. Empirical Musicology Review.

Peters, D. P., \& Ceci, S. J. (1982). Peer-review practices of psychological journals - the fate of accepted, published articles, submitted again. Behavioral and Brain Sciences, 5(2): 187-195. 